\title{
Selection of Reference Axis for Distal Femoral Rotation in TKA: A Literature Review
}

\author{
Ravikant Kamal* \\ H.No 2710, DLF Phase-4, Gurgaon, 122002, India \\ Received 12 May 2018, Accepted 14 July 2018, Available online 17 July 2018, Vol.8, No.4 (July/Aug 2018)
}

\begin{abstract}
Total knee arthroplasty (TKA) is widely accepted and standard surgical procedure for osteoarthritis. Most patients are able to recover their preoperative pain completely and achieve a good range of motions to perform daily tasks after TKA. However, there is a number of patients who remained unsatisfied with the result after TKA and often complain pain in performing daily task. Exploring reason behind post-operative pain and failure of TKA; researchers had found that in 31.2\% of these cases; failure is due to aseptic loosening. Aseptic loosening is closely related to improper component placement. Orienting component in the axial direction is critical for proper outcome of TKA. Measured resection and gap balancing are two methods used for rotational alignment of component. Studies have shown that the measured resection technique has an advantage over gap balancing. However, the outcome of measured resection technique depends on the proper selection of reference axis. Transepicondyle axis, Whiteside line and Posterior condyle line are mostly used for setting the component rotation in TEA. All three rotational axis has its own advantages and pitfalls, therefore selection of reference should be based upon knee bone morphology and diseased condition. Malalignment of component placement in the axial plane may cause patella maltracking, anterior knee pain, aseptic loosening and also it could be one of the reasons behind TKA revision. The Success of rotational alignment depends upon proper preoperative planning and accurately selection of landmarks involved in creating axis. In this review paper, Pros and cons of reference axis are discussed individually and their correlations with each other are explored based on existing literature.
\end{abstract}

Keywords: The Total Knee Replacement, Distal Femoral Rotation, Transepicondyle Line, Whiteside Line, Posterior Condyle line.

\section{Introduction}

The number of TKA surgeries is increasing every year. According to the study done by Inacio et al, 69\% of increment in IR (incidence rate) may be expected by 2050 compared to 2012(M.Inacio et al, 2017). Advancement of technology\& survival rate of implants has increased significantly which clearly reflects an increasing number of young patients opting for TKA.

In spite of all the advancements from manufacturer side, post-operative patient satisfaction rate has not increased in same proportion (R.Lau et al, 2012). Component misalignment is still a foremost challenge in TKA which has been considered one of the reasons behind TKA failure (R.E Windsor et al, 1989). It has been seen as possible reason for patella femoral complications and anterior knee pain post operatively (R. L Barrack et al, 2001). There are two methods exist to correct the axial alignment of knee: Measured

*Corresponding author is an Independent Researcher-Orthopedic (ORCID ID: 0000-0001-8744-0620)

DOI: https://doi.org/10.14741/ijcet/v.8.4.17 resection technique and balanced gap technique. Balanced gap technique uses proximal tibial resection as a reference for femoral axial alignment. Balancer will be used prior to the 4 in 1 cut to check the ligament stiffness. In case of stiffness on either side of knee, first ligament release will be done and it will be ensured that equal balance is achieved in either side.

Presence of severe deformity in tibia can lead the rotational alignment in varus and also the excessive release of ligament can cause patella tracking problem in balanced gap method. Comparatively, measured resection technique may be more useful in these cases (H. Hanada et al, 2007).Considering measured resection technique; rotational references are used for restoring the native rotational alignment in TKA. Three established references: Transepicondylar axis; Whiteside line \& Posterior condylar axis are used for rotational measurement in TKA (R.A Siston et al, 2005). Literature has shown that there are no clear advantages of one reference over the other two. However, it was suggested to pick the reference based on its reproducibility and deformity present in the individual patient. 
Pre-op and Post-op measurement techniques also play a vital role in execution and evaluation of rotational alignment. Rotational accuracy in different surgical method - Conventional, PSI and Navigation varies significantly. Regardless of surgical method, selection of references is critical for better rotational alignment of TKA. It is also important not to generalize rotational alignment as there is enough evidence in the literature which supports individualization rotational alignment in TKA.

\section{References used for rotational alignment}

Axial positioning of the femoral component is referred as rotational alignment. Any misalignment of rotational component may affect the patella tracking and can cause flexion instability. Femoral malalignment could be a possible cause of anterior knee pain (D. Shervin et al, 2015). Predicting distal femoral rotation may be challenging using X-ray modality as the landmarks are not very clear in X-ray. CT scan modality may be the better option for determining reference axis for rotation.

\subsection{Transepicondylar axis}

It is further divided into two parts- Clinical TEA and Surgical TEA. Surgical TEA is defined as the line joining the medial sulcus and lateral epicondyle point where else clinical or anatomical TEA is defined as the line joining the apex of medial prominence and lateral epicondyle (H.C Shon et al, 2012). Surgical TEA is mostly used as a rotational reference for TKA during the surgery. It is considered as the functional axis around which the knee rotates during flexion arc (T. Asano et al, 2005). However, Mochizuki has found there is a considerable amount of vertical displacement in medial epicondyle during 0 to $90^{\circ}$ of flexion. He has found the vertical displacement of $7.6 \mathrm{~mm}$ in medial side. He has counter the hypothesis as TEA being functional axis through this study. In his opinion, If TEA is considered as functional flexion axis (FFA) then there should not be any vertical displacement during the flexion range (T.Mochizuki et al, 2014). Determining epicondyle landmark intra-operatively may be challenging. It depends on user's experience as an experienced surgeon might pick landmark with better reproducibility rate than new surgeons. Epicondyle region is covered with thick soft tissue and its anatomical variation is evident. (C. Yan et al, 2008). According to the study done by Jean Yves Jenny et al. on 20 knees with 2 senior surgeons using Navigation, he found high variation with mean variability was $5^{\circ}$ for surgeon- 1 and $6^{\circ}$ for surgeon-2 (Jean Yves Jenny et al, 2004). Many of times presence of hardware or deformity in epicondyle region may make it more difficult to produce TEA.

\subsection{Whiteside line}

Whiteside line is defined as the line joining the lowest point of patella groove and top of inter-condylar notch.
Its intraoperative reproducibility and accuracy as a rotational reference are comparatively lesser than TEA (W.Yau et al, 2008). Although comparing visibility of landmark, Whiteside line has better visibility over TEA but presence of osteophyte in intercondylar region may create some problem in identifying the Whiteside line. It can be also be used as the cross-check to see the patella remains in neutral position through the flexion arc. Nicolas et al performed a study to check inter and intraobserver reliability of Whiteside's line using Navigation system. He found good reliability as compared to predefined Whiteside's line using Navigation. Whiteside's line was $2.1 \pm 1.5$ externally rotated compared to Navigation. There was no significant difference between experienced and new surgeons in terms of accuracy of landmark picking (N. Vanin et al, 2011). In cases of hyperplasia where posterior-lateral condyle is abnormally smaller, Whiteside line was suggested to be used as a reference (M. Akagi et al, 1999).In case of trochlear dysplasia or destructive arthritis, Whiteside line may fail to give proper alignment (F. Paternostre et al 2014).

\subsection{Posterior condylar line}

Posterior condyle line (PCL) is defined as a tangential line joining the prominent point of posterior-medial and posterior-lateral condyles and angle between posterior condyle line and transepicondyle line is called as posterior condyle angle (PCA). PCL is one of the most popular references for rotational alignment. However, in case of hypoplasia cases, it is not good option to opt for PCL for rotational alignment. The underdeveloped condyle may give incorrect PCA value which can cause femoral malalignment. The posterior condylar is also prone region for floating osteophyte. According to Frankie et al average posterior condylar angle was found $3.7 \pm 2.2^{\circ}$ and there was no significant difference between male and female patient. The angle varied based on diseased condition, In case of varus 3.3 $\pm 1.9^{\circ}$ and case of valgus, it was $5.4 \pm 2.3^{\circ}$ (F. Griffin et al, 1998). Increase in PCA angle from native PCA angle can tilt HKA angle in varus direction and decreasing PCA angle can tilt HKA angle in valgus direction during the motion of extension to flexion (Z. Zhao et al, 2015). Accuracy-wise it is least reproducible reference comparing to TEA \& Whiteside line but most of the manufacturer still uses PCL as a reference for implant design as it is easier to setup rotation using PCA and sizing can be done simultaneously (C.C. Castelli et al, 2016). The normal procedure for achieving ideal alignment starts with placing AP sizer on the distally resected surface and then providing $3^{\circ}$ external rotation in order to make it parallel to TEA. Plenty of studies have shown flaw in generalization of external rotation and supported individualization of rotation as per case (A. Gurava Reddy et al, 2016).

\subsection{Alternative references}

As every reference used earlier has some flaw, researchers have looked upon new references and 
landmarks as an alternative option for placing component on the axial plane. Simon Talbot et al has introduced the concept of sulcus line. He defined sulcus line as a 3D landmark which is a curve derived from multiple points selected in trochlear groove unlike to Whiteside line where only two points were taken to construct the axis as deepest point of patella groove in anterior direction and intercondylar notch at knee center region. He assessed SL line in 200 knees and shown the result that SL line was more accurate in comparison of Whiteside line (S.Talbot et al, 2015). He also suggested that anterior surface near trochlear could be also used for reference since it is a flat surface and chance of anatomical variation is less (S.Talbot et al, 2008).

\subsection{Correlation between references}

Although there are plenty of references available for femur; none of the references can give complete accuracy in femoral rotation. It is advisable to use more than one reference to overcome the limitation of each axis (F. Middleton et al, 2007). Frederic et al also suggested that combination of PCL and Whiteside line could be a reliable landmark of axial alignment (F.Paternostre et al, 2014). Another interesting study pitched the idea of averaging sulcus line and PCL and used it for femoral rotational alignment, Tat woon chao et al had shown the result using sulcus line and PCL may reduce malrotation (T.W. Chao et al, 2017).

Preoperative MRI based study done by fabricio also suggested that anatomical transepicondyle line is easiest axis to reconstruct with the accuracy of $30.8 \%$ of variation, surgical epicondyle variation was noted to $69 \%$ and the accuracy of Whiteside line $58.7 \%$. It is possible that landmark picked on 2D scan preoperatively and landmark picked in clinical setup may show significant variations. Robert et al performed study including 11 orthopedic surgeons with 10 cadaver specimen. He included 5 axis in which the reference axis was taken from navigation system and evaluated accuracy of rest of axis. He found only $17 \%$ of knee where rotated less than $5^{\circ}$ taking reference of digitized epicondyle axis(R.A. Siston et al, 2005).In the study done by W.P Yau et al, 56\% of TEA, $72 \%$ of PCL, $60 \%$ of Whiteside line was found in outlier range which supports high variability in all axis.

The variation in references may also depend on surgical approach used for TKA. W.P Yau et al performed a cadaver study in which he compared minimal invasive and conventional approach and found the error of TEA was more in minimal approach than the conventional approach. However, he found that Whiteside line error was less in minimally invasive approach than conventional approach (W. Yau et al, 2008).

\subsection{Population-based variation}

As bone morphology varies across the geographical region, it has a direct impact on the measurement of
PCA. Gurva Reddy et al have done the study on Indian Population and calculated average PCA value, he found only $27.7 \%$ of Indian population consists of PCA with $3^{\circ}$ (A. Gurava Reddy et al, 2016). Performing Iranian population with 108 patients, Jabalameli et al. found the mean value between anatomical transepicondyle and PCA was $5.9 \pm 1.6^{\circ}$ and the angle between clinical epicondyle and PCA was $1.6 \pm 1.7^{\circ}$ (M. Jabalameli et al, 2016)

\section{Discussion}

Exploring literature it is now evident that Transepicondyle line has comparatively better accuracy over Whiteside line and PCA (C.J. McDougall et al, 2016) but there is enough doubt involved to rely upon on one reference, hence it would be better option to actively use preoperative planning with multiple references and then reach into the conclusion on the most suitable reference for individual case. Importance of selecting proper rotational alignment is critical for TKA. Plenty of studies exists which shows misalignment in the rotation could cause anterior knee pain and patella dislocation, stiffness in retinaculam ligament \& osteonecrosis of patella (K.M Ghosh et al, 2010).

Preoperative and Postoperative radiological evaluations are also important to compare the outcome properly. Particularly in axial rotation X-ray image modality may not give an accurate result. CT based measurement is better option. Even post-op CT based images consist of artifacts and picking up landmarks would be challenging at times. Konigsberg et al have performed a study to determine inter and intraobserver variability in 2D landmarks picked postoperatively at the time of revision. He found that there is considerable inter-observer variability in femoral rotation where else intra-observer variability was good for femoral rotation (B. Konigsberg et al, 2014).In the comparative study done by Kanekasu between X-ray and CT scan shows a high correlation between both modality and suggested that X-ray could be used for rotational assessment as it is less costly and can give an equivalent result. X-ray was captured at $90^{\circ}$ flexed position to make it equivalent to flexion position (K. Kanekasu et al, 2005).

\section{Conflict of interest}

No benefits in any form have been received or will be received from a commercial party related directly or indirectly to the subject of this article.

\section{References}

Akagi, M., Matsusue, Y., Mata, T., Asada, Y., Horiguchi, M., Lida, H., \& Nakamura, T. (1999), Effect of Rotational Alignment On Patellar Tracking in Total Knee Arthroplasty. Clinical Orthopedic and Related Research, (366),155-63.

Asano, T., Akagi, M., \& Nakamura, T. (2005), The Functional Flexion-Extension Axis of the Knee Corresponds to the 
Surgical Epicondylar Axis, The Journal of Arthroplasty, 20(8),1060-7.

Barrack, R. L., Schrader, T., Bertot, A. J., Wolfe, M. W., \& Myers, L. (2001). Component rotation and anterior knee pain after total knee arthroplasty, Clinical orthopedic and related research , (392),48-55.

Castelli, C. C., Falvo, D. A., Iapicca, M. L., \& Gotti, V. (2016), Rotational alignment of the femoral component in total knee arthroplasty. Annals of Translational Medicine, $4(1), 4$.

Chao, T. W., Geraghty, L., Dimitriou, P., \& Talbot, S. ( (2017) ), Averaging rotational landmarks during total knee arthroplasty reduces component malrotation caused by femoral asymmetry, Journal of Orthopaedic Surgery and Research, 12,74.

Ghosh, K. M., Merican, A. M., Iranpour, F., Deehan, D. J., \& Amis, A. A. (2010), The Effect of Femoral Component Rotation on the Extensor Retinaculum, Journal of orthopedic research , 1136-41.

Griffin, F. M., Insall, J. N., \& Scuderi, G. R. (1998), The Posterior Condylar Angle in Osteoarthritic Knees, The journal of arthroplasty , 13(7),812-5.

Gurava Reddy, A., Mathur, R., Mugalur, A., Eachempati, K., \& Reddy, A. (2016), Reference axes for optimal femoral rotational alignment in varus osteoarthritic Indian knees A CT based study. Journal of clinical orthopaedic and trauma, 7(Suppl 2), 215-219.

Hanada, H., Whiteside, L., Steiger, J., Dyer, P., \& Naito, M. (2007), Bone Landmarks Are More Reliable Than Tensioned Gaps in TKA Component Alignment, Clinical Orthopedic and Related Research, 462,137-42.

Inacio, M., Paxton, E., Graves, S., Namba, R., \& Nemes, S. (2017), Projected increase in total knee arthroplasty in the United States - an alternative projection model. Osteoarthritis and Cartilage, 1797-1803.

Jabalameli, M., Moradi, A., Bagherifard, A., Radi, M., \& Mokhtari, T. (2016), Evaluation of Distal Femoral Rotational Alignment with Spiral CT Scan before Total Knee Arthroplasty (A Study in Iranian population), The Archive of Bone and Joint Surgery, 4(2), 122-127.

Jenny, J.-Y., \& Boeri, C. (2004), Low reproducibility of the intra-operative measurement of the transepicondylar axis during total knee, Acta Orthop Scand, 75 (1), 74-77.

Kanekasu, K., Kondo, M., \& Kadoya, Y. (2005), Axial radiography of the distal femur to assess rotational alignment in total knee arthroplasty, Clinical Orthopedic and Related Research , (434),193-7.

Konigsberg, B., Hess, R., Hartman, C., Smith, L., \& Garvin, K. L. (2014), Inter- and Intraobserver Reliability of Twodimensional CT Scan for Total Knee Arthroplasty Component Malrotation. Clinical Orthopedic and Related Research, 472(1), 212-217.

Lau, R., Gandhi, R., Mahomed, S., \& Mahomed, N. (2012), Patient satisfaction after total knee and hip arthroplasty. Clinics of geriatric medicine, 28(3),349-65.

McDougall, C. J., Gallie, P., \& Whitehouse, S. L. (2016), Preoperative assessment of femoral rotation and its relationship with coronal alignment: A magnetic resonance imaging study, Journal of orthopedic, 13(4), 290-293.
Middleton, F., \& Palmer, S. (2007), How accurate is Whiteside's line as a reference axis intotal knee arthroplasty? The Knee, 14(3),204-7.

Mochizuki, T., Sato, T., Blaha, J. D., Tanifuji, O., Kobayashi, K., Yamagiwa, H., Endo, N. (2014), The clinical epicondylar axis is not the functional flexion axisof the human knee, Journal of orthopedic science, 19(3),451-6.

Paternostre, F., Schwab, P., \& Thienpont, E. (2014), The combined Whiteside's and posterior condylar line as a reliable reference to describe axial distal femoral anatomy in patient-specific instrument planning, Knee Surg Sports Traumatol Arthrosc, 22(12),3054-9.

Shervin, D., Pratt, K., Healey, T., Nguyen, S., Mihalko, W. M., ElOthman, M. M., \& Saleh, K. J. (2015), Anterior knee pain following primary total knee arthroplasty, World Journal of Orthopedic, 6(10), 795-803.

Shon, H.C., Choi, E.S., Kim, Y.M., Kim, D.S., Park, K.J., Cho, B.K., Kim, S.W. (2012), Femoral Component Rotation of Total Knee Arthroplasty in Korean Subjects: A Study Using Grand Piano Sign and Computed Tomography, Journal of Korean Orthopedic Assosiation, 47(6), 432-438.

Siston, R. A., Patel, J. J., Goodman, S. B., Delp, S. L., \& Giori, N. J. (2005), The Variability of Femoral Rotational Alignmentin Total Knee Arthroplasty, The journal of bone and joint surgery, 2276-80.

Talbot, S., \& Bartlett, J. (2008), The anterior surface of the femur as a new landmark for femoral component rotation in total knee arthroplasty, Knee Surg Sports Traumatol Arthrosc, 16,258-262.

Talbot, S., Dimitriou, P., Radic, R., Zordan, R., \& Bartlett, J. (2015), The sulcus line of the trochlear groove is more accurate than Whiteside's Line in determining femoral component rotation, Knee Surg Sports Traumatol Arthrosc., 23(11), 3306-3316.

Vanin, N., Panzica, P., Dikos, G., Krettek, C., \& Hankemeier, S. (2011), Rotational alignment in total knee arthroplasty: intraoperative inter- and intraobserver reliability of Whiteside's line, Archives of Orthopaedic and Trauma Surgery, 131, 1477.

Victor, J., Doninck, D. V., Labey, L., Glabbeek, F. V., Parizel, P., \& Bellemans, J. (2009), A common reference frame for describing rotation of the distal femur, The bone \& Joint Journal, 91(5),683-90.

Windsor, R. E., Giles, R. S., Moran, M. C., \& Insall, J. N. (1989), Mechanisms of Failure of the Femoral and Tibial components in Total Knee Arthroplasty, Clinical orthopedic and related research, (248),15-9.

Yan, C., Yau, W., Ng, T., Lie, W., \& Tang, W. ( 2008), Inter- and intra-observer errors in identifyingthe transepicondylar axis and Whiteside's line, Journal of Orthopaedic Surgery, 16(3),316-20.

Yau, W., Leung, A., Liu, K., Yan, C., Wong, L., \& Chiu, K. (2008), Errors in the identification of the transepicondylar and anteroposterior axes of the transepicondylar and anteroposterior axes of the minimally-invasive and conventional approaches, The journal of bone and joint surgery, 90(4),520-6.

Zhao, Z., Wang, W., Wang, S., Jiang, L., Zhang, S., \& Zhao, Y. (2015), Femoral rotation influences dynamic alignmentof the lower extremity in total knee arthroplasty, International Orthopaedics (SICOT), 39,55-60. 